\title{
Automated Map Generation for an Intelligent Routing System
}

\author{
Muhammed Shefeek \\ Computer Science and Engineering, ICET, Mahatma Gandhi University, Muvattupuzha, Kerala, India
}

\begin{abstract}
The vehicle routing problem (VRP) is a combinatorial optimization and integer programming problem seeking to service a number of customers with a fleet of vehicles. VRP is an important problem in the fields of transportation, distribution and logistics. Often the context is that of delivering goods located at a central depot to customers who have placed orders for such goods. Implicit is the goal of minimizing the cost of distributing the goods. Many methods have been developed for searching for good solutions to the problem, but for all but the smallest problems, finding global minimum for the cost function is computationally complex. This paper aims at providing a real time solution to the travelling salesman problem based on Ant Colony Optimization (ACO) algorithm.
\end{abstract}

Keywords: VRP, Optimization, Travelling Salesman Problem, ACO.

\section{Introduction}

The traveling salesperson (or, salesman) problem (TSP) is a well-known and important combinatorial optimization problem. The goal is to find the shortest tour that visits each city in a given list exactly once and then returns to the starting city. Despite this simple problem statement, solving the TSP is difficult since it belongs to the class of NPcomplete problems. The importance of the TSP arises besides from its theoretical appeal from the variety of its applications. Typical applications [3] in operations research include vehicle routing, computer wiring, cutting wallpaper and job sequencing. The main application in statistics is combinatorial data analysis, e.g., reordering rows and columns of data matrices or identifying clusters.

The Vehicle Routing Problem (VRP) dates back to the end of the fifties of the last century when Dantzig and Ramser set the mathematical programming formulation and algorithmic approach to solve the problem of delivering gasoline to service stations. Since then the interest in VRP evolved from a small group of mathematicians to the broad range of researchers and practitioners, from different disciplines, involved in this field today.

The VRP definition states that $m$ vehicles initially located at a depot are to deliver discrete quantities of goods to $n$ customers. Determining the optimal route used by a group of vehicles when serving a group of users represents a VRP problem. The objective is to minimize the overall transportation cost. The solution of the classical VRP problem is a set of routes which all begin and end in the depot, and which satisfies the constraint that all the customers are served only once. The transportation cost can be improved by reducing the total travelled distance and by reducing the number of the required vehicles.

The majority of the real world problems are often much more complex than the classical VRP. Therefore in practice, the classical VRP problem is augmented by constraints, such as vehicle capacity or time interval in which each customer has to be served, revealing the Capacitated Vehicle Routing Problem (CVRP) and the Vehicle Routing Problem with
Time Windows (VRPTW), respectively. Here we adopt Capacitated Vehicle Routing Problem (CVRP).

This paper aims at providing a real time solution to the travelling salesman problem. Here we are going to implement a system where we are able to find out the best possible path for a delivery network .Here we use Google maps. In the map first we specify our warehouse is. Warehouse is the location from which we will begin the delivery network. Then we specify the clients in the map. There can be any number of clients. Each client has different requirement weightages. This requirement weightages are in addition to the normal travelling salesman problem. We can add vehicles with different capacities. We will be delivering the requirements using these vehicles. Once everything is set, the clients, vehicles and the warehouse we can calculate the paths for each vehicle for maximum efficient paths with specified vehicles.

\section{Related Works}

\subsection{Travelling Salesman Problem}

The traveling salesperson problem is a well known and important combinatorial optimization problem. The goal is to find the shortest tour that visits each city in a given list exactly once and then returns to the starting city. Formally, the TSP can be stated as follows. The distances between $n$ cities are stored in a distance matrix $D$ with elements $d_{i j}$ where $i, j=1 \ldots . n$ and the diagonal elements $d_{i i}$ are zero. A tour can be represented by a cyclic permutation $\pi$ of $\{1$, $2 \ldots n\}$ where $\pi$ (i) represents the city that follows city $i$ on the tour. The traveling salesperson problem is then the optimization problem to find a permutation $\pi$ that minimizes the length of the tour denoted by

$$
\sum_{i=1}^{n}=d_{i \pi(i)}
$$

The NP-completeness of the TSP already makes it more time efficient for small-to-medium size TSP instances to rely on heuristics in case a good but not necessarily optimal solution 


\section{International Journal of Science and Research (IJSR) \\ ISSN (Online): 2319-7064 \\ Index Copernicus Value (2013): 6.14 | Impact Factor (2014): 5.611}

is sufficient. In TSP, the term distance is used interchangeably with dissimilarity or cost and, unless explicitly stated, no restrictions to measures which obey the triangle inequality are made.

TSP heuristics typically fall into two groups, tour construction heuristics which create tours from scratch and tour improvement heuristics which use simple local search heuristics to improve existing tours. The implemented tour construction heuristics are the nearest neighbor algorithm and the insertion algorithms.

The nearest neighbor algorithm follows a very simple greedy procedure. The algorithm starts with a tour containing a randomly chosen city and then always adds to the last city in the tour the nearest not yet visited city. The algorithm stops when all cities are on the tour. An extension to this algorithm is to repeat it with each city as the starting point and then return the best tour found. This heuristic is called repetitive nearest neighbor.

All insertion algorithms start with a tour consisting of an arbitrary city and then choose in each step a city k not yet on the tour. This city is inserted into the existing tour between two consecutive cities $\mathrm{i}$ and $\mathrm{j}$, such that the insertion cost (i.e., the increase in the tour's length)

$$
d(i, k)+d(k, j)-d(i, j)
$$

is minimized. The algorithms stop when all cities are on the tour. The insertion algorithms differ in the way the city to be inserted next is chosen. Some of the variations are,

Nearest insertion:-The city $\mathrm{k}$ is chosen in each step as the city which is nearest to a city on the tour.

Farthest insertion: - The city $\mathrm{k}$ is chosen in each step as the city which is farthest from any of the cities on the tour.

Cheapest insertion: - The city $\mathrm{k}$ is chosen in each step such that the cost of inserting the new city is minimal.

Arbitrary insertion: - The city $\mathrm{k}$ is chosen randomly from all cities not yet on the tour.

\subsection{TSP Variations}

Asymmetric and symmetric TSP: - An important distinction can be made between the symmetric TSP and the more general asymmetric TSP. For the symmetric case (normally referred to as just TSP), for all distances in D the equality $\mathrm{dij}=$ dji holds, i.e., it does not matter if we travel from $\mathrm{i}$ to $\mathrm{j}$ or the other way round, the distance is the same. In the asymmetric case (called ATSP), the distances are not equal for all pairs of cities. Problems of this kind arise when we do not deal with spatial distances between cities but, e.g., with the cost or necessary time associated with traveling between locations, where the price for the plane ticket between two cities may be different depending on which way we go. Solving the TSP is comparatively easier than the ATSP, since the symmetry reduces the number of feasible solutions to half.
The Generalized Traveling Salesman Problem (GTSP):-

The Generalized Traveling Salesman Problem (GTSP) is a variant of the standard Traveling Salesman Problem (TSP). As in the TSP, the graph considered consists of $n$ nodes, and the cost between any two nodes is known. The GTSP differs from the TSP in that the node set is partitioned into $\mathrm{m}$ clusters. An optimal GTSP solution is a cycle of minimal cost that visits exactly one node from each cluster.

\section{Travelling Purchaser Problem (TPP):-}

The Travelling Purchaser Problem (TPP) deals with a set of $m$ markets and a set of $p$ products that the salesman must purchase. Each product is available, although with different quantities, in a subset of markets. However, the price of each of the products depends on the market where it is available. The demands for each product and the cost of traveling are the givens of the problem. In TPP, the objective is to purchase the whole demand of products, departing and returning to a depot, so as to minimize the sum of the costs of travelling and of the products purchased .The applications of the TPP are mainly in the contexts of networking, routing and scheduling.

\section{Vehicle Routing Problem}

The globalization of the economy leads to a rapidly growing exchange of goods on our planet. Limited commodities and transportation resources, high planning complexity and the increasing cost pressure through the strong competition between logistics service providers make it essential to use computer-aided systems for the planning of the transports. An important subtask in this context is the operational planning of trucks or other specialized transportation vehicles. These optimization tasks are called Vehicle Routing Problems (VRP). The problem of finding optimal routes for groups of vehicles, the Vehicle Routing Problem (VRP), belongs to the class of NP-hard combinatorial problems [1]. The fundamental objectives are to find the minimal number of vehicles, the minimal travel time or the minimal costs of the travelled routes. In practice the basic formulation of the VRP problem is augmented by constraints such as e.g. vehicle capacity or time interval in which each customer has to be served, revealing the Capacitated Vehicle Routing Problem (CVRP) and the Vehicle Routing Problem with Time Windows (VRPTW) respectively. The real-world problems mostly encompass the capacity and time constraints.

\subsection{Constructive heuristics}

The first step of the heuristic VRP solving is the construction of a feasible initial solution. In the lucky case when handmade solution already exists, it can be considered as a substitution for the constructive heuristics. Generally, constructive heuristics follows the idea that customers are selected on some cost minimization criterion and routes are constructed matching capacity and time constrains. Methods with sequential approach construct one route at a time, while parallel methods build several routes simultaneously. Some constructive methods are two-phase methods and can be divided into two classes cluster first, route-second methods 


\section{International Journal of Science and Research (IJSR) \\ ISSN (Online): 2319-7064}

Index Copernicus Value (2013): 6.14 | Impact Factor (2014): 5.611

and route-first, cluster-second methods. In the first case, customers are first organized into feasible clusters, and a vehicle route is constructed for each of them. In the second case, a tour is first built on all customers and then segmented into feasible vehicle routes.

\subsubsection{Nearest Neighbour Heuristic for CVRP}

The Nearest Neighbour Heuristic (NNH) is a constructive method for generating initial feasible solution for CVRP with the simple idea of inserting the nearest neighbour of the last inserted customer in the route. The first inserted customer on the route can be selected randomly or with some arbitrary criteria like the farthest distance customer from the depot. From this seed route, every other customer is inserted by the criteria of the nearest neighbour from the last inserted customer until the capacity of the vehicle is exhausted according to the definition of the CVRP problem where every customer has its own demand for the delivery or pick up. This method is derived from Travelling Salesman Problem (TSP) heuristic approach.

\subsubsection{Nearest Addition Heuristic for CVRP}

The Nearest Addition Heuristic (NAH) is an extended version of $\mathrm{NNH}$ where one of the unserved customers is selected for insertion and added to the existing route between two already visited neighbours. The total price of insertion has to be the minimal value that is calculated by adding two new distances produced by linking of the unvisited customer with neighbour's and by subtracting the distance between the visited neighbour's customers in the selected route.

\subsubsection{Sweep Heuristic for CVRP}

One of the most known two-phase constructive methods for CVRP is the sweep algorithm. This is a two-phase algorithm that belongs to the cluster-first, route second methods. In the first phase the algorithm decomposes the CVRP problem by clustering customers in m-TSP problems. The customer clustering is conducted by two criteria. The positions of all customers are transformed in polar coordinates with the depot in the origin of the coordinate system. The first criterion for grouping customers is the minimal angle. The second criterion matches the capacity of the vehicle which is assigned to the cluster, so that the total demands of all the selected customers has to be less than or equal to the capacity of the vehicle. The first and the second criteria are combined so that the assignment of customers to groups is performed by increasing the angular coordinate from 0 to the value where capacity of the assigned vehicle for that cluster is exhausted. The last step optimizes each vehicle route (cluster) separately by solving the corresponding TSP.

\subsubsection{Clark and Wright Heuristic for CVRP}

This method is one of the first originally developed heuristics for CVRP and it is frequently used. The algorithm starts from the initial solution where each route has only one customer and a corresponding vehicle. At the start, the number of vehicles is equal to the number of customers. Every new iteration should reduce the number of vehicles unifying two routes that give maximal savings, e.g. reduction of overall distance or time. There are two variants of algorithm: one with sequential and other with parallel construction of routes. The parallel version yields better results.

\section{Automated Map Generation for an Intelligent Route} Allocation System

This paper aims at providing a real time solution to the travelling salesman problem. Here we are going to implement a system where we are able to find out the best possible path for a delivery network .Here we use Google maps. In the map first we specify our warehouse is. Warehouse is the location from which we will begin the delivery network. Then we specify the clients in the map. There can be any number of clients. Each client has different requirement weightages. This requirement weightages are in addition to the normal travelling salesman problem. We can add vehicles with different capacities. We will be delivering the requirements using these vehicles. Once everything is set the client, vehicles and the warehouse we can calculate the paths for each vehicle for maximum efficient paths with specified vehicles.

The most successful soft computing algorithm to obtain the optimal solution of the TSP is the Ant Colony Optimization (ACO) algorithm was first proposed by Dorgio [5]. The development of the ACO algorithm has been inspired by the foraging behaviour of some ant species. When foraging for food, the ants deposit pheromone on the ground in order to mark some favourable path that should be followed by other members of the colony. The ACO algorithm exploits a similar mechanism for solving optimization problems. Among the better performing ACO algorithms are variants such as Ant Colony System (ACS), MAX-MIN Ant System (MMAS), rank-based Ant System [4] and the cunning Ant System (cAS) [2].

Ant Colony Optimization (ACO) algorithm is a metaheuristic optimization technique that is used to minimize the cost and maximize the efficiency of an optimization problem. ACO uses four main parameters that are influence of pheromone on direction $(\alpha)$, influence of adjacent node distance $(\beta)$, pheromone evaporation coefficient $(\rho)$ and pheromone depositing factor $(\varsigma)$. This algorithm updates the trail on the basis of current pheromone density. Our intelligent route allocation system uses Ant Colony Optimization algorithm as the base for finding the efficient paths with specified vehicles.

\section{Conclusion}

This paper aims at providing a real time solution to the travelling salesman problem based on Ant Colony Optimization algorithm. Here we are going to implement a system where we are able to find out the best possible path for a delivery network .Here we use Google maps. In the map first we specify our warehouse is. Warehouse is the location from which we will begin the delivery network. Then we specify the clients in the map. Each client has different requirement weightages. This requirement weightages are in addition to the normal travelling salesman problem. We can add vehicles with different capacities. We will be delivering the requirements using these vehicles. Once everything is set the clients, vehicles and the warehouse we can calculate the 
paths for each vehicle for maximum efficient paths with specified vehicles.

\section{Acknowledgment}

The Author would like to thank Joyal Ulahannan, Assistant Professor, Department of Information Technology, Ilahia College of Engineering and Technology, Muvattupuzha for his moral and technical support.

\section{References}

[1] Ropke, S. \& Pisinger, D. (2006). “A unified heuristic for a large class of vehicle routing problems with backhauls." European Journal of Operational Research, Vol. 171, No. 3, June 2006, 750-775, ISSN: 0377-2217

[2] Tsutsui, S., cAS: Ant colony optimization with cunning ants, Proc. of the 9th Int. Conf. on Parallel Problem Solving from Nature (PPSN IX), 2006, pp. 162-171.

[3] Punnen A. "The Traveling Salesman Problem: Applications, Formulations and Variations."In Gutin and Punnen (2002), chapter 1, pp. $1\{28$.

[4] Bullnheimer, B., Hartl, R., \& Strauss, C. (1999). A new rank- based version of the Ant System: A computational study. Central European J Operations Res Econom.

[5] Dorigo, M., \& Caro, D. G. (1999).Ant colony optimization:a new meta- heuristic (vol. 2).Proceeding of the 1999 Congress on Evolutionary Computation.

\section{Author Profile}

Muhammed Shefeek received the Bachelor of Technology degree in Computer Science and Engineering from Mahatma Gandhi University, Kerala. He is currently doing Master of Technology degree in Computer Science and Engineering with Specialization in Information Systems from Mahatma Gandhi University, Kerala. 